\title{
Application of piezoelectric fiber composite actuator to aircraft wing for aerodynamic performance improvement
}

\author{
LI Min ${ }^{1}$, YUAN JunXian ${ }^{1}$, GUAN De ${ }^{1} \&$ CHEN WeiMin ${ }^{2 *}$ \\ ${ }^{1}$ School of Aeronautics Science and Engineering, Beihang University, Beijing 100191, China; \\ ${ }^{2}$ Key Laboratory of Environmental Mechanics, Institute of Mechanics, Chinese Academy of Sciences, Beijing 100190, China
}

Received August 9, 2010; accepted October 15, 2010

\begin{abstract}
The application of actuator made of piezoelectric material, particularly the advanced piezoelectric fiber composite due to the rapid development of smart materials and structures and active control technology in aviation and aerospace industry, to aircraft for performance enhancements such as flight control, aerodynamic force optimization, structure weight reduction, and overall aircraft design represents a new challenge to researches. It is considered as one of the key technologies for developing future flight vehicle. An approach with virtual control surface instead of conventional control surface to control aerodynamic force distribution and flight performance by use of piezoelectric fiber composite actuators distributed on wing surface is presented here. Particularly, the design and implementation of increasing lift force, providing roll maneuver, decreasing induced drag and wing root moment in different flight environments by the same structure control platform are studied. The control effect and sensitivity are examined quantitatively. Generally speaking, better control effect can be obtained by making better use of aeroelastic character to enlarge the actuation strain produced by piezoelectric material.
\end{abstract}

piezoelectric actuator, piezoelectric fiber composite, shape control, aerodynamic characteristics, morphing wing

Citation: Li M, Yuan J X, Guan D, et al. Application of piezoelectric fiber composite actuator to aircraft wing for aerodynamic performance improvement. Sci China Tech Sci, 2011, 54: 395-402, doi: 10.1007/s11431-010-4212-0

\section{Introduction}

In recent decades smart materials and structures, such as piezoelectric actuator, have been currently used in structure control because of their high density of energy, wide range of response frequencies, good linear feature, flexible location and direct loading [1]. The researches performed in application of aviation and aerospace have been mainly focused on dynamic control and static control [2]: (1) vibration and noise control, e.g. buffet control in F/A-18, flutter suppression to enlarge flight envelope, vibration control of engine and active noise control of aircraft; (2) shape control, e.g. shaping the wing of aircraft to improve aerodynamic

*Corresponding author (email: wmchen@imech.ac.cn) performance, self-adapting micro-vehicle and control of vortex wake. And consequently large varieties of piezoelectric actuation devices and smart structures have been developed $[3,4]$. Among the broad kinds of smart materials and structures, the piezoelectric composite actuation devices [5-8] benefiting from its higher power of actuating, directional nature of actuation, better flexibility and credibility, are becoming a basic control cell in practical aviation and aerospace fields. In fact, with the improvement of commercial performance piezoelectric fiber composite combined with carbon fiber composite has been applied to many projects such as morphing wing for long-endurance unmanned aircraft [9-11].

Morphing wing has interest to flight vehicle designer because of its advantages compared with the conventional wing. First, the morphing wing changes its shape to maxi- 
mize aircraft performance in significantly different environments unlike the conventional wing that is optimized only for a certain environment and its performance descends in other environments. A morphing aircraft, being capable of adapting its shape to generate the optimized performance when environments are varied, is thought to be the final object pursued by flight vehicle designers [12]. Second, the complexity of structure design and the structure weight can be significantly reduced by avoiding control surface and complex servo control system in conventional wing. Moreover, a direct aeroelastic effect, by which the added aerodynamic load provided by variation of wing shape is efficiently used to control flight attitude, can be applied. Whereas for a conventional wing the control reversal usually occurs and is generally unwelcome. It means that the aircraft structure design will return back to material strength rather than stiffness, or material efficiency can be increased and advantages of composite material can be fully used.

Among the presented researches, the shape controls of morphing wing based on larger geometry deformation, such as varying wing area, aspect ratio and sweep angle as well as the relevant studies on aerodynamic performance, control approach, stability and dynamic response, have been mainly addressed though by now not yet a definite structure configuration has been popularly acknowledged. However the researches, based on smaller or medium deformations and its subsequent added aerodynamic load resulting from the aeroleastic effect to improve the synthesis aerodynamic performance, are rarely seen. The work concerned with structure design, control method and studies on aerodynamic performance, dynamic and aeroelastic characteristics is required to be developed.

A control platform of actively shaping wing is presented in this paper. Distributed actuators made of piezoelectric fiber composite (PFC) are bonded on wing surface. Several control goals, i.e. increasing lift force, providing roll maneuver, decreasing induced drag and root moment in different flight environments, are reached by the same structure control platform. Additionally, the control effects of different fiber tailor configurations under corresponding flight conditions are examined on a scaled model. The approach and its quantitative results of sensitivity analysis provide original investigation for further application of PFC actuator to practical engineering.

\section{Mechanism and governing equations of aerodynamic force control}

For a conventional wing, aerodynamic force distribution is adjusted by a suitable deflection of leading (or trailing) edge of control surface. However, the control efficiency becomes lower when the aeroelastic effect becomes significant and unfortunately unwelcome aeroelastic phenomena, e.g. the aileron effect and the control reversal of control surface, occur. Consequently, higher stiffness of wing host structure is required and flight envelope is limited to smaller range. An approach with virtual control surface where actuators are made of piezoelectric fiber composite instead of traditional control surface (aileron or flap) has been proposed to shape airfoil and to produce added aerodynamic force by the enlarged strain due to aeroelastic effect. Furthermore, with this approach self-adaptive distributions of aerodynamic force (or moment) can be generated corresponding to different flight environments. So the flight attitude can be appropriately controlled. The mechanism of this approach can be described by the following equation of static aeroelastisity:

$$
\alpha_{\mathrm{f}}=\alpha_{0}+\alpha_{\mathrm{A}}+\alpha_{\mathrm{V}},
$$

where $\alpha_{0}$ and $\alpha_{\mathrm{f}}$ denote the initial and final angles of attack, $\alpha_{\mathrm{A}}$ is the angle produced by aerodynamic force and written as

$$
\boldsymbol{\alpha}_{\mathrm{A}}=q \boldsymbol{C} \boldsymbol{A} \boldsymbol{\alpha}_{\mathrm{f}},
$$

where $q$ is the dynamic pressure, $\boldsymbol{C}$ is the structure flexibility matrix, $c_{i j}$ donates stream-wise angle at downwash point of the $i$ th aerodynamic panel due to vertical load at pressure point of the $j$ th aerodynamic panel, and it can be given by FEM analysis and interpolation. $\boldsymbol{A}$ is the aerodynamic coefficients matrix, $a_{i j}$ denotes lift divided by $q$ on the $i$ th panel due to unit angle of attack at the $j$ th panel, and it can be given by vortex lattice method. $\alpha_{\mathrm{V}}$ is the angle of attack caused by actuating voltage. Then $\alpha_{\mathrm{f}}$ can be written as

$$
\boldsymbol{\alpha}_{\mathrm{f}}=(1-q \boldsymbol{C A})^{-1}\left(\boldsymbol{\alpha}_{0}+\boldsymbol{\alpha}_{\mathrm{V}}\right) .
$$

The aerodynamic force distributed on the wing surface is

$$
\boldsymbol{F}_{\mathrm{A}}=q \boldsymbol{R} \boldsymbol{A} \boldsymbol{\alpha}_{\mathrm{f}}=q \boldsymbol{R} \boldsymbol{A}(1-q \boldsymbol{C A})^{-1}\left(\boldsymbol{\alpha}_{0}+\boldsymbol{\alpha}_{\mathrm{v}}\right),
$$

where $\boldsymbol{R}$ is a row vector, if $\boldsymbol{R}=1, \boldsymbol{X}, \boldsymbol{Y}$, where $1, \boldsymbol{X}, \boldsymbol{Y}$ represent unit row vector, $x$ coordinate row vector and $y$ coordinate row vector of aerodynamic pressure points, respectively, then $\boldsymbol{F}_{\mathrm{A}}$ are the lift force, aerodynamic moments about $y$ axis and $x$ axis, respectively.

For a conventional wing, $\alpha_{\mathrm{V}}=0$ in eq. (4), so the aerodynamic force (or moment) is produced by changing the values of the corresponding elements which belong to control surface in matrix $\boldsymbol{\alpha}_{0}$. However, for a wing with virtual control surface the aerodynamic force (or moment) is produced by changing element values of $\boldsymbol{\alpha}_{\mathrm{v}}$. The main mechanism differences of controls for the two wings are as follows:

(1) On the view of the control effects, the aeroelastic effect exhibits significantly different influences. For a conventional wing the direction of lift force increment produced by deflection of control surface is often opposite to the direction of the lift force increment on host wing surface. Therefore the control effect becomes lower and can even reach to an unwelcome level, the control reversal, as the 
aeroelastic effect increasing. Whereas for a wing with virtual control surface that wing behaves as a whole control surface, the value of lift force increment increases as the aeroelastic effect increasing. So no control reversal below the dynamic pressure of divergence exists. And the stiffness requirement for host wing surface can be reduced.

(2) On the view of the deformation forms produced, an arbitrary airfoil deformation can be generated by piezoelectric actuators flexibly distributed on the overall wing surface to satisfy aerodynamic requirement. Whereas only the deflection at leading (or trailing) edge is generated by conventional control surface. Compared with the leading (or trailing) edge control surface, the virtual control surface, that total wing surface can be deformed as a larger control surface, has a higher order of magnitude of control area. So smaller deformation is capable of reaching a higher level of control.

(3) On the view of the distribution of aerodynamic force, the aerodynamic force singularity and the subsequent calculation difficulties are successfully avoided for the virtual control surface. Since unlike the conventional wing the virtual control surface does not have a gap between the control surface and the host wing surface, the aerodynamic force is continuous and its distribution is smoother.

In addition, two useful points can be inferred from eq. (4). First, effects of $\alpha_{0}$ and $\alpha_{\mathrm{V}}$ can be linearly superposed, and $\boldsymbol{F}_{\mathrm{A}}$ is linearly proportional to $\alpha_{0}$ and $\alpha_{\mathrm{V}}$ equally. So ignoring the equal influence of $\boldsymbol{\alpha}_{0}$, only the $\boldsymbol{F}_{\mathrm{A}}$ produced by $\boldsymbol{\alpha}_{\mathrm{V}}$ will be considered in numerical simulations below. Second, $\boldsymbol{F}_{\mathrm{A}}$ has different nonlinear relationships with $q$ (indicating flight condition), $\boldsymbol{C}$ (indicating influence of structure stiffness and position of stiff axis) and $\boldsymbol{A}$ (indicating geometry and main parameters of aerodynamic surface). In this paper the control mechanism and effect are explored on a simplified model simulating a high aspect ratio, unswept wing. The influences of $q$ and $\boldsymbol{C}$ on $\boldsymbol{F}_{\mathrm{A}}$ are mainly addressed here. Other factors such as geometry and parameters of aerodynamic surface are not included, since they belong to the applicability of virtual control surface to varied flight vehicles and will be investigated in other work.

\section{Classes and actuation effects of piezoelectric actuators}

From eq. (4) one can see that piezoelectric actuator is mainly used to produce an appropriate $\boldsymbol{\alpha}_{\mathrm{V}}$ which is the distributed angle of attack and it is proportional to the simulated load $M_{\mathrm{V}}$ produced by the control voltage. In the range of linear geometry, the control effect mainly results from the torsion deformation of airfoil rather than the bending deformation, because the torsion efficiency of bending deformation is low. For example, although a torsion effect can be generated by two bending with reverse direction respectively at leading edge and trailing edge, the subsequent an- gle of attack is very small [13]. Among piezoelectric actuators, the one made of PFC is most suitable to airfoil control for the following reasons:

(1) Compared with isotropic piezoelectric ceramic such as PZT5H, PFC with fiber oriented in $45^{\circ}$ direction is capable of producing the maximum shear stress and consequently the maximum torsion deformation and stream-wise angle when equal voltage is applied to it [14].

(2) Researches on MFC (Macro Fiber Composite, an advanced version of PFC), have been mature. Large variety of researches on MFC's fabrication, installment, usage and fatigue life have been done in recent years. Moreover, series products of MFC have been commercial for practical engineering.

(3) To designer of practical aircraft wing skin, it is acceptable and convenient to use PFC layer instead of conventional carbon fiber composite layer without changing wing internal structure. It is perhaps the approach very first realized in practical engineering.

For torsion actuation, effective layer configurations might be assorted into three classes according to fiber direction combined with direction of the applied electric field [15]: 1) fibers are symmetrically aligned on top and bottom surfaces of wing in $+45^{\circ}$ angle direction and electric fields in two opposite directions are applied ( called $+45^{\circ} \mathrm{SA}$ below); 2) fibers are symmetrically aligned in $-45^{\circ}$ angle direction and electric fields in opposite directions are applied (called $-45^{\circ} \mathrm{SA}$ below); 3 ) fibers are anti-symmetrically aligned in $\pm 45^{\circ}$ angle direction and electric fields in the same direction are applied (called $\pm 45^{\circ} \mathrm{AS}$ below). The three modes were selected because they could give remarkable stream-wise angle. The effect and reason were discussed in ref. [14]. It is noted that $+45^{\circ} \mathrm{SA}$ and $-45^{\circ} \mathrm{SA}$ produce the same deformations. However the two configurations have different levels of the aeroelastic effect because of different relative positions between the structure stiff axis and aerodynamic pressure center. So we select these three layer configurations. The simulated actuation moment producing $\alpha_{\mathrm{V}}$ for three configurations can be written as [14]

$$
M_{\mathrm{v}}=\sigma_{\mathrm{v}} l t_{\mathrm{p}}\left(t_{\mathrm{p}}+t\right),
$$

where $t$ and $t_{\mathrm{p}}$ are the thickness of the thin plate and piezoelectric sheet respectively, $\sigma_{\mathrm{V}}$ is the simulated actuation stress depending on material elastic constant $c_{\mathrm{ij}}$, piezoelectric constant $d_{i j}$ and electric field strength $E_{i}, l$ is the length (or width) of the piezoelectric sheet.

\section{Control of aerodynamic performance by changing airfoil shape}

The illustrative model shown in Figure 1(a) has a structure coordinate system consistent with the aerodynamic coordinate system in which $x$ axis parallels direction of flow ve- 
locity $v$, wing surface is in $x$-y plane. Model parameters come from a high-altitude and long-endurance unmanned aircraft [9]. The basic similarity ratios are length $K_{1}=1 / 50$, density $K_{\rho}=1 / 1$, dynamic pressure $K_{\mathrm{q}}=1 / 1$, derived similarity ratios is stiffness $K_{\mathrm{k}}=1 / 50^{4}$. The geometry size and location of piezoelectric actuator are explored, so the aspect ratio of analysis model is 14:1. The scaled model has a size of $l$ (length) $\times b($ width $) \times t$ (thickness $)=560 \mathrm{~mm} \times 80 \mathrm{~mm} \times 1.5 \mathrm{~mm}$ and aerodynamic meshes as 20 (span) $\times 4$ (chord). The actuator is made of F1 MFC (series No. M8557F1 [8] with properties listed in Table 1 and manufactured by SMC) that belongs to $45^{\circ}$ torsion actuator. The effective geometry size of one actuator is $85 \mathrm{~mm} \times 57 \mathrm{~mm} \times 0.3 \mathrm{~mm}$ and the total geometry size including manufacturing frame is $110 \mathrm{~mm} \times 75$ $\mathrm{mm} \times 0.3 \mathrm{~mm}$. 5 sheets of MFC are bonded respectively on the top and bottom surfaces of wing. Three configurations, i.e. $+45^{\circ} \mathrm{SA},-45^{\circ} \mathrm{SA}$ and $\pm 45^{\circ} \mathrm{AS}$, are considered here. Our control approach is aimed at four objects, i.e. increasing lift force, providing roll maneuver, decreasing the induced drag and decreasing wing root moment in a typical case with cruise dynamic pressure $1600 \mathrm{~Pa}$. As a comparison, a model with conventional aileron (shown in Figure 1(b)) is also analyzed, its parameters including geometry, stiffness and layer configurations (with MFC actuators but no voltage application) are the same with model in Figure 1(a) except that it has two ailerons (outside and inside) with an area of $25 \%$ wing area.

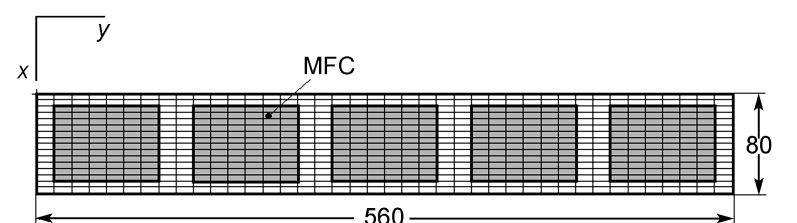

(a)

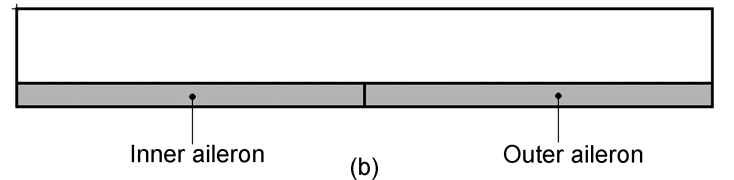

Figure 1 Sketch of the scaled wing model.

Table 1 Geometric and material property parameters of MFC provided by SMC [8]

\begin{tabular}{cc} 
Size $(\mathrm{mm})$ & $85 \times 57 \times 0.3$ \\
Low-field $(|E|<1 \mathrm{kV} / \mathrm{mm})$, unbiased-operation piezoelectric constants \\
$d_{33}{ }^{\text {a) }}\left(\times 10^{-12} \mathrm{~m} / \mathrm{V}\right)$ & 400 \\
$d_{31}{ }^{\mathrm{b})}\left(\times 10^{-12} \mathrm{~m} / \mathrm{V}\right)$ & -170 \\
Orthotropic linear elastic properties (constant electric field) \\
Tensile modulus, $E_{1}{ }^{\mathrm{a})}(\mathrm{GPa})$ & 30.34 \\
Tensile modulus, $E_{2}{ }^{\mathrm{b})}(\mathrm{GPa})$ & 15.86 \\
Poisson's ratio, $v_{12}$ & 0.31 \\
Poisson's ratio, $v_{21}$ & 0.16 \\
Shear modulus, $G_{12}{ }^{\mathrm{c}}(\mathrm{GPa})$ & 5.52 \\
\hline
\end{tabular}

a) Fiber-direction; b) electric direction; c) rules-of-mixture estimate.
Optimization technology is required for the four control objects. Here the $N$ dimensions extremum plural optimization method under restriction conditions is employed. We have found that when classical difference-oriented optimization methods, e.g. the steepest descent method, utility function and feasible direction method, are used the overall optimization point is sometimes missed. And the optimization efficiency declines for problem with multi-variables, like the control problems in this paper. In addition, concerning the practical characters of material property and engineering structure, the constraint condition for the optimization problem is set as a constraint for the actuation electric field strength, i.e. $E \leqslant 1000 \mathrm{~V} / \mathrm{mm}$.

\subsection{Increasing lift}

As we know, the symmetrically added lift produced by $\boldsymbol{\alpha}_{\mathrm{V}}$ is capable of controlling aircraft pitch maneuver. The added lift respectively produced by the five couples (consisting of actuators respectively on top surface and bottom surface of wing) of MFC actuators has the same sensitivity sign though their magnitude values are different. So the values of electric field strength applied on each couple of MFC actuators are equally set to be $1000 \mathrm{~V} / \mathrm{mm}$. The plots of the non-dimensional lift $L_{\mathrm{V}} / L_{\mathrm{C}}$ versus the dynamic pressure $q$, where $L_{\mathrm{V}}$ and $L_{\mathrm{C}}$ are the added lift produced by actuation voltage and $1^{\circ}$ deflection of aileron respectively, are shown in Figure 2. The plots show that: 1) MFC actuator can generate an added lift effect similar to the effect by aileron, that is at $1600 \mathrm{~Pa}$ dynamic pressure the added lift by a single MFC layer is equal to that by $5.8^{\circ}-10.2^{\circ}$ deflection of aileron; 2) efficiencies of three layer configurations are different and the differences continue to increase with dynamic pressure.

Although the rise of the added lift with dynamic pressure may be explained by aeroelastic effect, it is interesting to investigate the differences between three layer configurations. Therefore, $\alpha_{\mathrm{V}}$ and $\alpha_{\mathrm{f}}$ at mid-chord for three layer con-

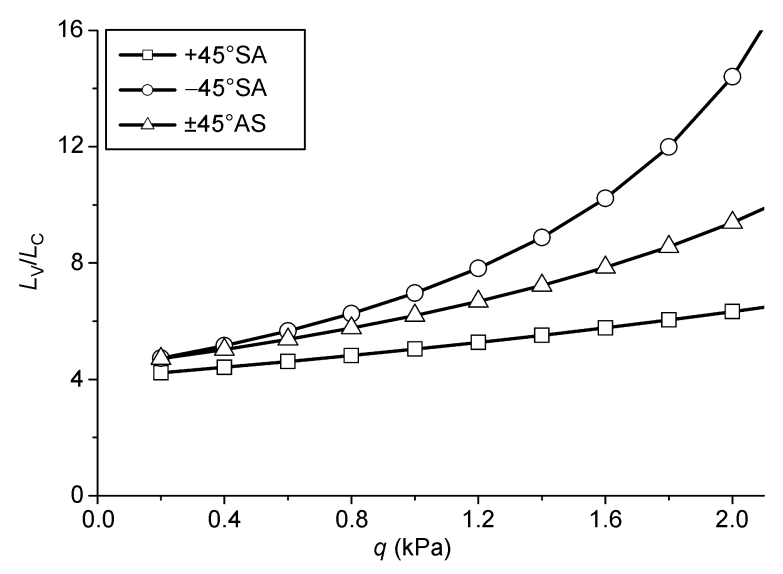

Figure 2 Non-dimensional lift $L_{\mathrm{V}} / L_{\mathrm{C}}$ versus the dynamic pressure $q$. 
figurations are compared in Figure 3. We note that in Figure 3(a) no obvious difference between $\alpha_{\mathrm{V}}$ and even an approximate coincidence between plot of $+45^{\circ} \mathrm{SA}$ and plot of $-45^{\circ} \mathrm{SA}$ are seen. Whereas obvious differences between $\alpha_{\mathrm{f}}$, the final angle of attack involving influence of aeroelasticity, are seen in Figure 3(b). The reason causing the difference of $\alpha_{\mathrm{f}}$ is mainly that different layer configurations have different positions of structure stiff axis. Subsequently, distances between the stiff axis and aerodynamic pressure center are different for the three configurations, while the distance is critical to aeroelastic effect. For example, in $+45^{\circ} \mathrm{SA}$ configuration the structural stiff axis is moved forward and consequently aeroelastic influence is decreased, inversely in $-45^{\circ} \mathrm{SA}$ configuration the stiff axis is moved backward and consequently aeroelastic influence is maximized. Moreover, the relative positions of stiff axis for three configurations are plotted in Figure 4 where $x_{\mathrm{r}}$ is the stiff center coordinates of the cross section and $2\left(x_{\mathrm{r}}-b / 2\right) / b$ indicates the distance between the stiff center and the middle-chord of the wing. The variation of stiff axis position is also responsible for different values of diverge velocity of wing, i.e. $\infty$ (for $+45^{\circ} \mathrm{SA}$ ), $2212 \mathrm{~Pa}$ (for $-45^{\circ} \mathrm{SA}$ ) and $3548 \mathrm{~Pa}$ (for $\pm 45^{\circ} \mathrm{AS}$ ) respectively.

In addition, comparing values of attack angle in Figure 3(a) with values in Figure 3(b), one may be aware of the
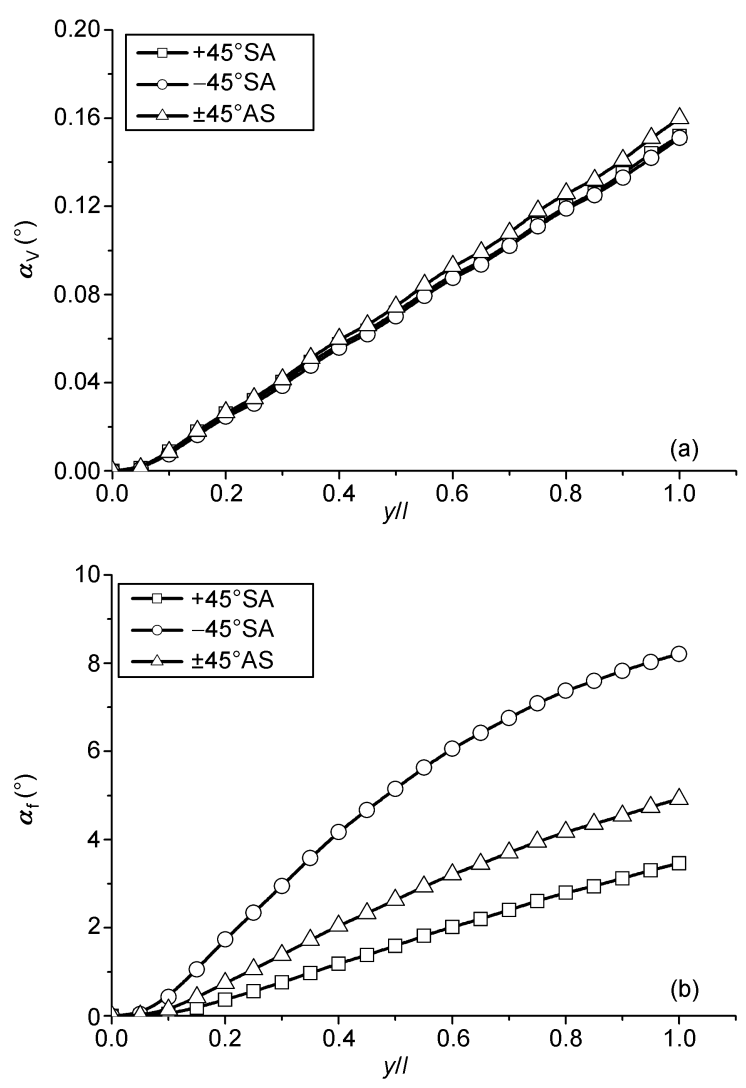

Figure 3 Distributions of $\boldsymbol{\alpha}_{\mathrm{V}}$ and $\boldsymbol{\alpha}_{\mathrm{f}}$ for different layer configurations. (a) Distributions of the mid-chord $\alpha_{\mathrm{V}}$ along the wing span; (b) distributions of the mid-chord $\alpha_{\mathrm{f}}$ along the wing span.

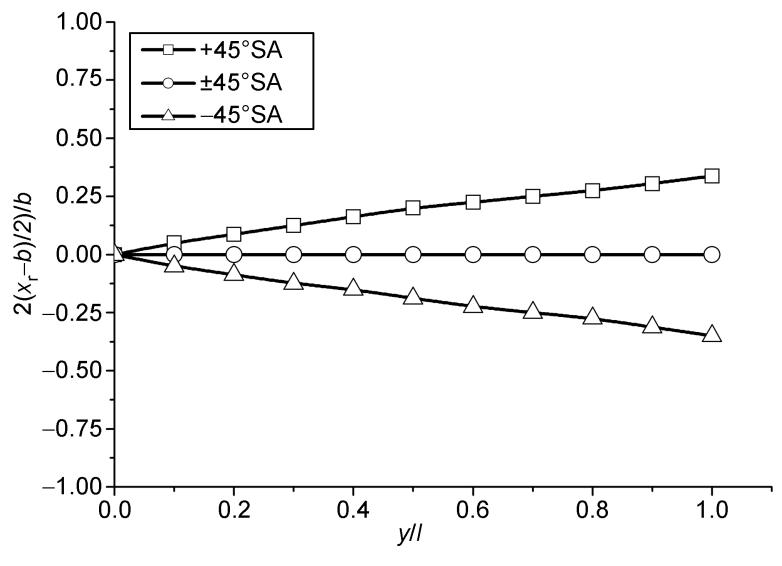

Figure 4 Positions of the elastic axis for different layer configurations.

significant effect resulting from the aeroelasticity phenomenon. The value of $\boldsymbol{\alpha}_{\mathrm{f}}$ enlarged by aeroelastic effect is much greater than the value of $\alpha_{\mathrm{V}}$ produced by actuator itself by times of several orders. In Figure 3 it is around 20-50 times at the typical dynamic pressure. By the aeroelastic effect enlarging small deformation to generate the added aerodynamic force and further to control overall aircraft flight attitude, an interesting effect of small input resulting in large output is realized here.

\subsection{Improving roll performance}

The roll rate is used to describe the roll performance of aircraft as

$$
p=M_{x} / M_{x, p=1},
$$

where $M_{x}$ indicates the roll moment produced by $\alpha_{\mathrm{V}}$ or $\boldsymbol{\alpha}_{0}=[0, \boldsymbol{\delta}]^{\mathrm{T}}, \boldsymbol{\delta}$ is the deflection angle of aileron. $M_{x, p=1}$ indicates the roll damping moment when $p=1 \mathrm{deg} / \mathrm{s}$, the initial angle of attack $\boldsymbol{\alpha}_{0}=\boldsymbol{Y} / v$ and $\boldsymbol{\alpha}_{\mathrm{V}}=0, v$ is flight velocity. Similar to aileron deflection, on the condition of the anti-symmetric aerodynamic $\boldsymbol{\alpha}_{\mathrm{V}}$ produces an added moment $M_{x}$ so as to control the roll motion of aircraft. The applied electric field strength on each couple of MFC actuators is $1000 \mathrm{~V} / \mathrm{mm}$ same as the value in Section 4.1, since the roll moments produced by the five couples of MFC actuators respectively have the same sensitivity sign. The non-dimensional roll ratio $p_{\mathrm{V}} / p_{\mathrm{C}}$ versus the dynamic pressure $q$, where $p_{\mathrm{V}}$ and $p_{\mathrm{C}}$ are the roll moment produced by the actuation voltage and 1 deflection of outside aileron respectively, are presented in Figure 5.

Figure 5 shows that: 1) MFC actuator similar to aileron can generate a roll effect, and at $1600 \mathrm{~Pa}$ dynamic pressure the roll ratio produced by a single MFC layer is equal to that by $7.7^{\circ}-8.2^{\circ}$ deflection of outside aileron; 2) roll rate increases with dynamic pressure while the differences of their values of the three layer configurations are not as obvious as the differences of lift force in Figure 2. In fact similar to the 


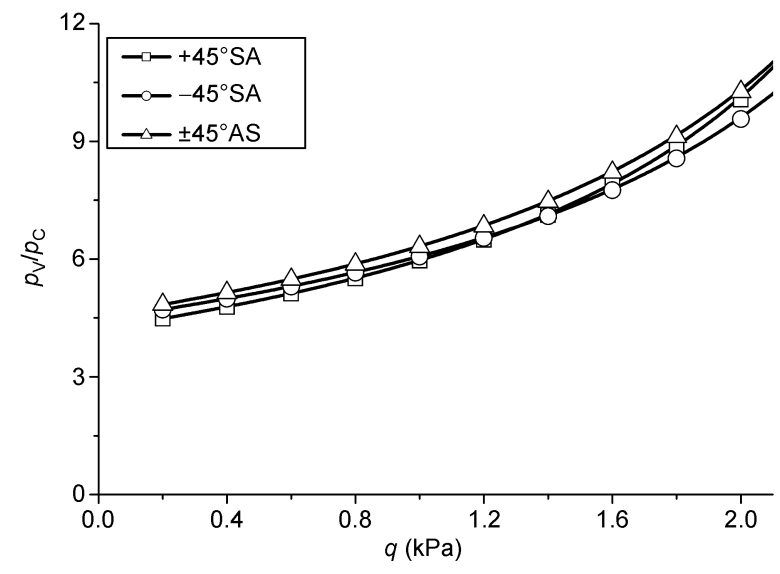

Figure 5 Non-dimensional roll ratio $p_{\mathrm{v}} / p_{\mathrm{C}}$ versus the dynamic pressure $q$.

lift force, the value of roll moment $M_{x}$ really depends on the layer configurations, so does the corresponding damping moment $M_{x, p=1}$. Thus the final values of the roll rate, the ratio of $M_{x}$ to $M_{x, p=1}$, for three configurations are nearly not varied, as seen in Figure 5, when the layer configurations are varied.

\subsection{Decreasing the induced drag force}

The induced drag is one of the key components of aerodynamic drag, its decrease benefits long-endurance or heavy-loading of aircraft. We take $\boldsymbol{\alpha}_{0}=1^{\circ}$ accompanied with the actuation voltage being zero as the basic case. Then values of the induced drag coefficient $C_{\mathrm{D}}$, lift force coefficient $C_{\mathrm{L}}$ and then $C_{\mathrm{D}} / C_{\mathrm{L}}^{2}[16]$ at a series of dynamic pressure are calculated by vortex lattice method in symmetric condition. Further, the actuation voltages are applied and the initial angles of attack $\alpha_{0}$ are adjusted by optimization technology so as to obtain the minimum value of $C_{\mathrm{D}}^{\mathrm{V}} / C_{\mathrm{L}}^{2}$ while the total lift force is maintained constant. The relative variation rate of the drag coefficient $e_{\mathrm{D}}=\left(C_{\mathrm{D}} / C_{\mathrm{L}}^{2}-C_{\mathrm{D}}^{\mathrm{V}} / C_{\mathrm{L}}^{2}\right) /$ $\left(C_{\mathrm{D}} / C_{\mathrm{L}}^{2}\right)$ versus the dynamic pressure and the electric field distribution are presented in Figures 6 and 7 respectively. In order to exhibit the detail of the control effect, the distribution of the lift force $L_{\mathrm{i}} / L$ respectively with and without the actuation voltage, where $L_{\mathrm{i}}$ and $L$ represent the lift forces on each span panel and on the total wing respectively, are presented in Figure 8.

In Figure 6 a 12.1\%-22.1\% reduction of the induced drag at the typical dynamic pressure is seen. Figure 7 and especially Figure 8 indicate that changing the span distribution of the lift force by the actuation voltages to approach to an expected shape is critical to the control of drag force. Three configurations generate nearly similar lift distributions where the maximum lift force occurs at around $20 \%$ wing span and lift similarly drops as approaching two span ends of the wing, though the lift distributions are different before

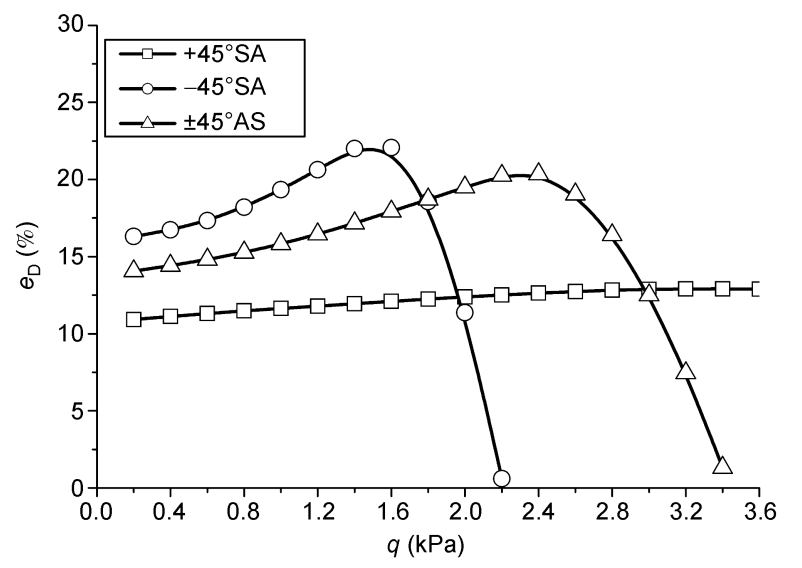

Figure 6 Drag coefficient $e_{\mathrm{D}}$ versus the dynamic pressure $q$.

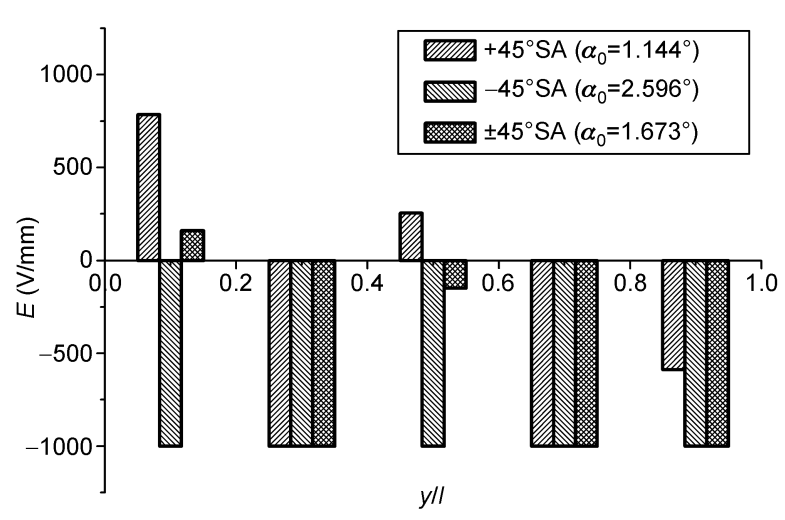

Figure 7 Distributions of the electric field $E\left(\alpha_{0}\right.$ means the initial angle of attack adjusted by optimization calculations).

the actuation voltages are applied.

The differences between the control effects of three configurations (in Figure 8) may be caused by two reasons. First, notwithstanding the steadiness of values of $\alpha_{\mathrm{V}}$ the values of $\boldsymbol{\alpha}_{\mathrm{f}}$ are varied obviously when the layer configurations are varied. As in Figure 3, the control effect of $-45^{\circ} \mathrm{SA}$ is the most remarkable. Second, as the dynamic pressure approaching the divergence value, effect of the actuation voltage significantly decreases. Because the maximum value of the actuation voltage is limited by the output level of the practical power supply, i.e. in reality the actuation power is finite, though $\alpha_{0}$ and $\alpha_{\mathrm{v}}$ play equal roles in providing aerodynamic forces, as shown in eq. (4). Consequently, not depending on the actuation voltage the control effect of $\boldsymbol{\alpha}_{0}$ on aerodynamic force rises rapidly compared to the effect of $\boldsymbol{\alpha}_{\mathrm{v}}$. This can be seen in Figure 6 where the control efficiencies drop respectively from $22.1 \%$ (1600 $\mathrm{Pa})$ to $0.6 \%(2200 \mathrm{~Pa})$ for $-45^{\circ} \mathrm{SA}$ (2212 $\mathrm{Pa}$ divergence dynamic pressure) and from $19.1 \%$ (2600 Pa) to $1.3 \%$ (3400 $\mathrm{Pa}$ ) for $\pm 45^{\circ} \mathrm{AS}$ ( $3548 \mathrm{~Pa}$ divergence dynamic pressure). It is also noted that two curves of $-45^{\circ} \mathrm{SA}$ and $\pm 45^{\circ} \mathrm{AS}$ in Figure 6 will collapse nearly into one if the horizontal coor- 

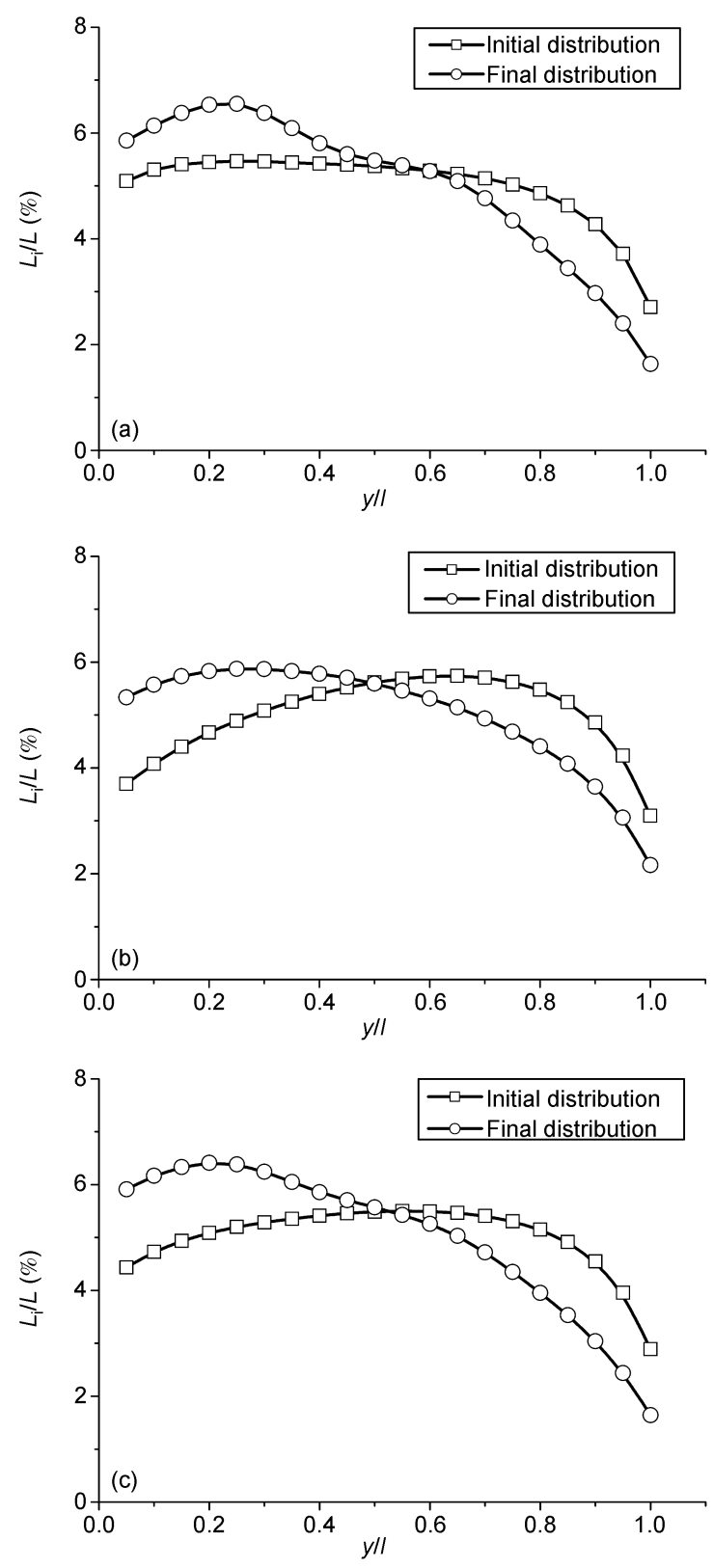

Figure 8 Variations of the lift force distributions before and after actuation voltages are applied on MFC actuators for control of reducing the induced drag force. (a) $+45^{\circ} \mathrm{SA}$; (b) $-45^{\circ} \mathrm{SA}$; (c) $\pm 45^{\circ} \mathrm{AS}$.

dinate is normalized by the corresponding divergence dynamic pressure.

\subsection{Reducing root bending moment}

The root bending moment $M_{x}$ of aircraft wing in the flight limit environment is a critical factor of wing structure strength design. Hence a strengthened structure, accompanied with the punishments of heavier weight and lower strength efficient (lower working stress) in most other flight conditions, has to be designed to satisfy the particular safety requirement for the flight limit notwithstanding the rare occurrence of the flight limit in practical aviation. Recently, a novel concept is suggested [5,11] that if the root moment can be actively reduced when an aircraft enters the flight limit environment, aircraft structure is needed not to be strengthened and consequently the weight can be decreased. Here we try to realize the idea on our model. Like in the above section, the basic case is set as $\boldsymbol{\alpha}_{0}=1^{\circ}$ combined $0 \mathrm{~V}$ actuation voltage. The values of $M_{x}$ without the actuation voltage are calculated from the aerodynamic forces in a symmetric condition. Further, the actuation voltages are applied and the initial angles of attack $\boldsymbol{\alpha}_{0}$ are adjusted by an optimization process to give the values of $M_{x}^{\mathrm{V}}$ while the total lift force maintains constant.

The relative variation rates of root moment $e_{\mathrm{M}}=\left(M_{x}-\right.$ $\left.M_{x}^{\mathrm{v}}\right) / M_{x}$ versus the dynamic pressure are presented in Figure 9 where a $10.7 \%-26.2 \%$ reduction of the root moment is observed. In addition, the span distributions of lift force $L_{\mathrm{i}} / L$ are presented in Figure 10. It shows that similar to the change rule of the distributions in Figure 8 the final $L_{\mathrm{i}} / L$ in Figure 9 monotonically drops as approaching the outer span end and meanwhile rises as approaching the inner span end compared to the initial distribution. Essentially, for problem of controlling root moment the object is to decrease the angle of attack at wing tip and meanwhile to increase the angle of attack at wing root as far as possible. But for problem of controlling the induced drag the object is to averagely adjust the lift forces. The optimized actuation voltage is the same with the voltage of $-45^{\circ} \mathrm{SA}$ in Figure 7. Generally speaking, the control effect (same as effect of $-45^{\circ} \mathrm{SA}$ in Figure 7) decreases with the dynamic pressure due to the limitation of the maximum control power. Therefore the control effect of $+45^{\circ} \mathrm{SA}$, where the structure stiff axis is nearer to the aerodynamic pressure center and the aeroelastic influence is weaker, is higher than effects of other configurations.

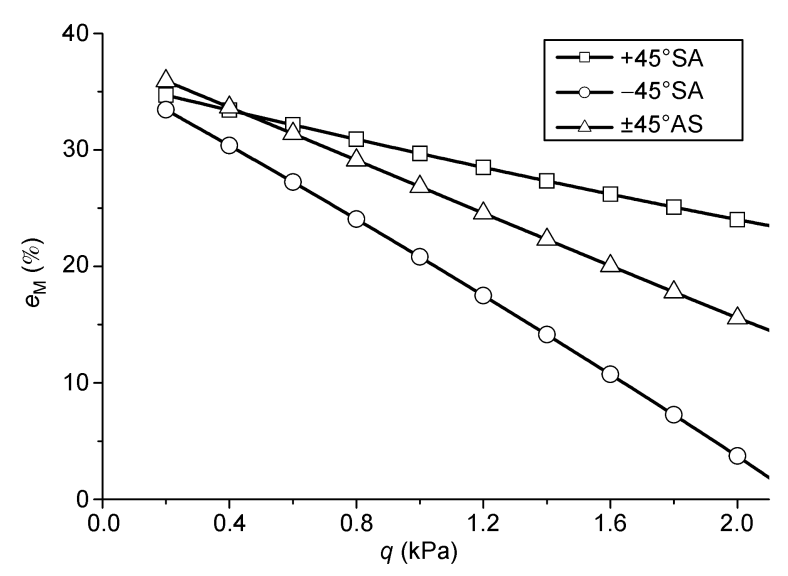

Figure 9 Relative variation rates of root moment $e_{\mathrm{M}}$ versus the dynamic pressure $q$. 

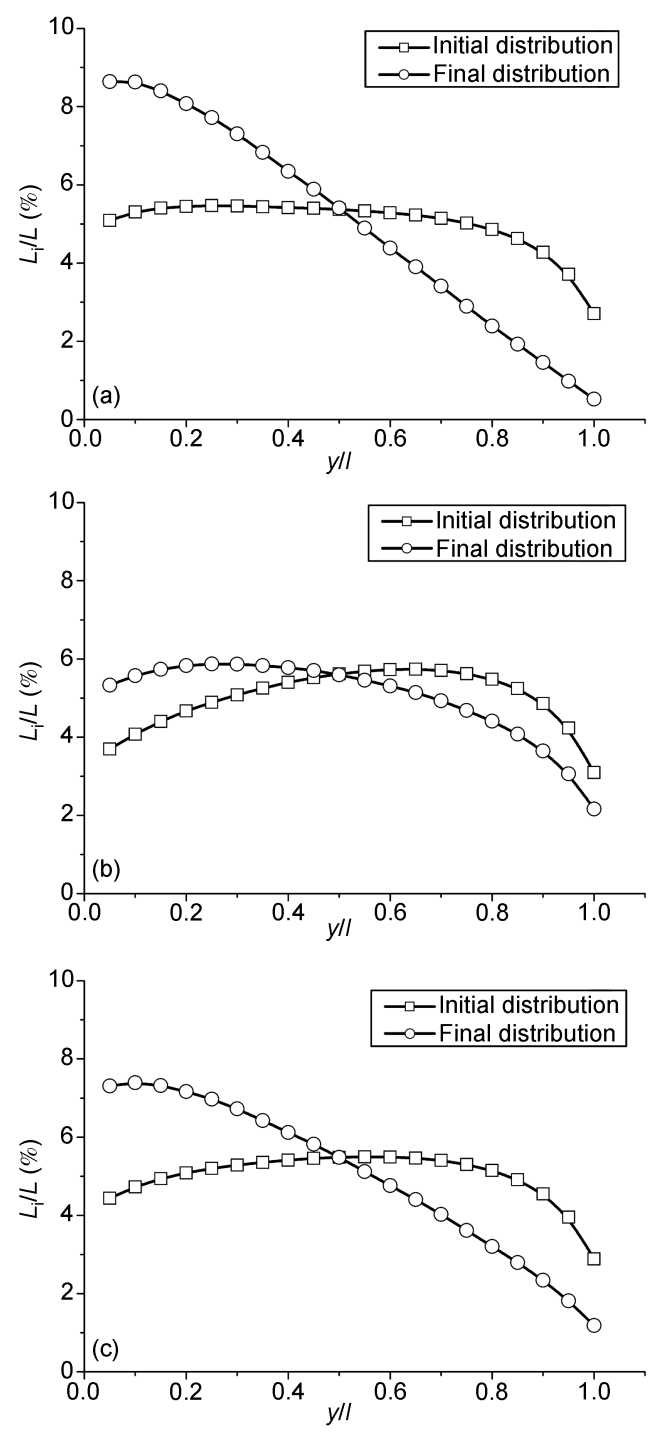

Figure 10 Variations of the lift force distributions before and after the actuation voltages are applied on MFC actuators for control of reducing the root bending moment. (a) $+45^{\circ} \mathrm{SA}$; (b) $-45^{\circ} \mathrm{SA}$; (c) $\pm 45^{\circ} \mathrm{AS}$.

\section{Conclusions}

The actuators, made of piezoelectric fiber composite and distributed on aircraft wing surface, are used to control the wing mechanical deformations. Our control objects are to enhance aircraft performances, such as to increase lift force, provide roll maneuver, decrease the induced drag and to decrease wing root moment in different flight environments. The control effects are examined and compared to the effect of a conventional control surface. By using MFC which has special torsion character, the control effects have been remarkably increased. Generally speaking, better control effect can be obtained by making better use of the aeroelastic characteristics to enlarge the actuation strain produced by PFC actuator. So our approach has more advantages over a flexible wing, e.g. a wing with higher aspect ratio, where an obvious aeroelastic phenomenon occurs and can be effi- ciently used to enhance aircraft performances.

On the side, some questions need to be explained. 1) Linear elastic material, small deformation and invariable piezoelectric constant hypothesis were used in this paper. 2) The MFC actuators were laid on the surface of the wing model. If the box model was used, the MFC actuators could be laid in wing rib structure to increase control effect. 3) Only monolayer MFC was used in the analysis model now, if multilayer MFC was used, the control effect would be remarkably increased but the influence between the electric field of multilayer MFC would be validated by calculation and test. 4) The drive effect test on the panel model with MFC has been accomplished, the maximum deviation between calculation and test is less than $5 \%$. The detail of test data and comparison between different calculation parameters would be discussed in other paper.

This work was supported by the Fundamental Research Funds for the Central Universities (Grant No. YWF-10-01-B05), the National Natural Science Foundation of China (Grant No. 10772183) and the Intellectual Innovation Project of the Chinese Academy of Sciences (Grant No. KJCX2-YW-L07)

1 Culshaw B. Smart Structures and Materials. Boston: Artech House, 1996

2 Yousefi-Koma A, Zimcik D G. Applications of smart structures to aircraft for performance enhancement. Canadian Aeronaut Space J, 2003, 49(4): 163-172

3 Niezrecki C, Brei D, Balakrishnan S, et al. Piezoelectric actuation: state of the art. Shock Vib Dig, 2001, 33(4): 269-280

4 Garcia E. Smart structures and actuators: past, present, and future. In: Proceedings of SPIE Conference on Smart Structures and Material Systems: Industrial and Commercial Applications of Smart Structures Technologies. Bellingham: SPIE, 2002. 1-12

5 Schultz M R, Hyer M W. A morphing concept based on unsymmetric composite laminates and piezoceramic MFC actuators. AIAA Paper 2004, 1806

6 Park J S, Kim J H. Material properties of single crystal macro fiber composite actuators for active twist rotor blades. AIAA Paper 2005, 2265

7 Williams R B. Nonlinear mechanical and actuation characterization of piezoceramic fiber composites. Doctoral Dissertation. Blacksburg: Virginia Polytechnic Institute and State University, 2004

8 SMC corp. http://www.smart-material.com, 2010, 9

9 Carlos E S C, Brown E L. Modeling of high aspect ratio active flexible wings for roll control. AIAA Paper 2002, 1719

10 Sahoo D, Carlos E S C. Roll maneuver control of UCAV wing using anisotropic piezoelectric actuators. AIAA Paper 2002, 1720

11 Bilgen O, Kochersberger K B, Inman D J. A novel aerodynamic vectoring control airfoil via macro-fiber-composite actuators plate airfoil designs. AIAA Paper 2008, 1700

12 Sanders B, Eastep F E, Forster E. Aerodynamic and aeroelastic characteristics of wings with conformal control surfaces for morphing aircraft. J Aircraft, 2003, 40(1): 94-99

13 Li M, Chen W M, Guan D. Improvement of aircraft rolling power by use of piezoelectric actuators. Chinese J Aeronaut, 2004, 17(2): 87-92

14 Li M, Chen W M, Wang M C, et al. A load simulation method of piezoelectric actuator in FEM for smart structures, Sci China Ser ETech Sci, 2009, 52(9): 2576-2584

15 Li M, Chen W M, Jia L J. Drive characteristics and stiffness influence with piezoelectric fiber composite actuators on airfoil surface. Chinese J Aeronaut, 2010, 31 (2): 418-425

16 Hedman S G. Vortex lattice method for calculation of quasi steady state loadings on thin elastic wings in subsonic flow. FFA Report 105, 1965 